\title{
TN14003 attenuates cartilage degeneration by targeted blocking of the SDF-1/CXCR4 signaling pathway in knee osteoarthritis
}

Yanlin Li

First Affiliated Hospital of Kunming Medical University

Kun Wang

First Affiliated Hospital of Kunming Medical University

Chuan He

First Affiliated Hospital of Kunming Medical University

Guoliang Wang

First Affiliated Hospital of Kunming Medical University

Guofeng Cai

First Affiliated Hospital of Kunming Medical University

Xianguang Yang

First Affiliated Hospital of Kunming Medical University

\section{En Song}

First Affiliated Hospital of Kunming Medical University

Rui Han ( $13529365266 @ 163 . c o m$ )

First Affiliated Hospital of Kunming Medical University

\section{Research Article}

Keywords: TN14003, SDF-1/CXCR4 signaling pathway, osteoarthritis, chondrocytes, cartilage degeneration

Posted Date: March 1st, 2021

DOl: https://doi.org/10.21203/rs.3.rs-270864/v1

License: (a) (i) This work is licensed under a Creative Commons Attribution 4.0 International License. Read Full License 


\section{Abstract}

In order to investigate the effect of TN14003 and its mechanism on cartilage degeneration in vitro and in vivo. P1 chondrocytes isolated from cartilage tissues of OA patients who underwent total knee arthroplasty were randomly assigned to blank control group, TN14003 group, T140 group, and AMD3100 group in vitro. Each group cells were cultured for 1, 2, 4, 6, 8, 10 days. Cell morphology were observed under inverted phase-contrast microscope and examined using MTT assay, flow cytometry, ELISA (MMPs in the chondrocyte medium) and quantitative real-time PCR (mRNA expressions of Col II and ACAN). Moreover, 96 male Hartley guinea pigs with spontaneous OA were randomly assigned to examine the effect of TN14003, T140, and AMD3100 in vivo. After 12 weeks, guinea pigs were sacrificed, the knee articular cartilage histopathology was analyzed. No difference in morphology, proliferation rate and apoptosis among four groups $(P>0.05)$. The content of MMP-3 and MMP-13, mRNA expression levels of ACAN and Col II were significantly lower in TN14003 group compared with other groups $(P<0.05)$. TN14003 had stronger effect in decreasing cartilage degeneration compared with T140 and AMD3100 in vivo. TN14003 could effective targeted to prevention and treatment of OA.

\section{Introduction}

Osteoarthritis $(\mathrm{OA})$ is one of the most common chronic degenerative joint diseases that cause pain and disability in adults. $O A$ is characterized by inflammation and extracellular matrix (ECM) degradation ${ }^{1,2}$. As the world population ages, the incidence of $O A$ is dramatically increasing year-by-year ${ }^{3-6}$. Symptomatic treatment is the main strategy for $\mathrm{OA}$, yet, so far, no therapy that could fully reverse or terminate the pathological process of OA has been developed ${ }^{1,7-11}$.

Recent studies have found that the SDF-1/CXCR4 signaling pathway has a pivotal role in the pathological process of cartilage degeneration in patients with $\mathrm{OA}^{12}$. CXCR4 antagonists can effectively block the SDF-1/CXCR4 signaling pathway and reduce the degeneration of articular cartilage in OA patients ${ }^{13-19}$. Until now, the FDA has approved few SDF-1/CXCR4 targeted antagonists; the most popular ones being AMD3100, T140, and TN14003. AMD3100 has been approved by the FDA for the treatment of HIV and tumors ${ }^{20-23}$. However, because of the flexible macrocyclic cyclam for the structure, it can bind to metal ions and form a highly stable metal chelate. In addition, this chelating property may lead to lung and liver fibrosis after long-term treatment ${ }^{24}$. AMD3100 is a partial antagonist of CXCR4, which has low antagonistic efficiency and high cytotoxicity ${ }^{25}$; its major disadvantages are cardiotoxicity, specific leukocytosis, and poor bioavailability of oral preparations ${ }^{26}$, which limit its clinical application.

T140 is a complete antagonist of CXCR4. Yet, T140 is not stable in feline serum due to the cleavage of the C-terminal Arg. Its biological stability is poor, and its antagonistic effect is not definite 25,27 .

TN14003 is a high-affinity CXCR4 antagonist composed of 14 amino acids, which is synthesized through $\mathrm{C}$-terminal amidation and $\mathrm{N}$-terminal 4-fluorobenzoyl modification. Its major advantages are stable chemical properties and low serum toxicity, and a wide range of drug concentrations ${ }^{27,28}$. In addition, 
TN14003 has better serum stability and lower cytotoxicity than T140; it can effectively block the SDF1/CXCR4 signaling pathway, and represent a safe, effective, and stable complete antagonist of the CXCR4 receptor. Currently, TN14003 is mainly used against tumor and HIV infection ${ }^{28-30}$, while only a few data are available regarding its application in OA-related cartilage degeneration.

Thus, the aim of this study was to investigate the effect of TN14003 and its underlying mechanism on cartilage degeneration in vivo and in vitro. The effect of TN14003 was compared to SDF-1/CXCR4 targeted antagonists, T140 group, and AMD3100.

\section{Results}

\section{The chondrocytes identification after digestion}

We have successfully isolated chondrocytes from articular cartilage of patients with osteoarthritis. After digestion, P1 chondrocytes (without the addition of drug) were in a latent growth phase for the first 3 days, after which they reached a logarithmic growth phase during days 4 and 8 and a plateau phase at day 10-12 (Fig. 1a, b). All cells were positive for toluidine blue staining (Fig. 1C), and Col II staining (Fig. 1d). Moreover, flow cytometry showed the early, middle, and advanced stages were < $5 \%$ (Fig. 2).

\section{Cell morphology after 10 days of drug intervention}

After 10 days of drug intervention, the morphological changes of chondrocytes in four groups were not significantly different. However, the degradation of type 2 collagen was obviously inhibited by TN14003 compared to other groups. Before drug intervention, cell morphology grew from small polygons and small circles to the fusiform shape. After 10 days of drug intervention, cells in the four groups showed spiral growth. The cell density and cell morphology were not significantly different between groups (Fig. 3 - 1). Also, the highest expression of Col II was observed in the TN14003 group, followed by the T140 group; the AMD3100 group was significantly lower than the T140 group ( $P<0.05$; Fig. $3-2)$. MTT assay indicated that there was no significant difference in cell proliferation between the four groups at the same time point (Fig. $<$ link rid="fig3">3</link $>-3$ ).

\section{Cell viability and apoptosis after 10 days of drug intervention}

As shown in Fig. $<$ link rid="fig3" $>3</$ link $>-3$, cells treated with the drug showed similar growth phases as those without drug intervention (see above). There was no significant difference in cell proliferation between the four groups at the same time point $(P>0.05)$. Moreover, flow cytometry showed no significant difference in the apoptotic rate among the four groups $(P>0.05 ;$ Fig. $4-1,4-2)$. These data suggested that drugs (TN14003, T140, AMD3100) at a concentration of $1000 \mathrm{nmol} / \mathrm{L}$ had no significant effect on the proliferation and apoptosis of OA chondrocytes. 


\section{The content of MMPs in the chondrocyte medium}

TN14003 can significantly slow down the release rate of MMP-3 and MMP-13 compared to other groups. The content of MMP-3 and MMP-13 decreased in a time-dependent manner in all groups, and at the same time point, the contents of MMP-3 and MMP-13 secreted into the medium by chondrocytes in the group of TN14003, T140, AMD3100, and blank control were successively decreased $(P<0.05)$. The MMP-3 and MMP-13 were significantly lower in the TN14003 group at all-time points compared to the other groups $(P<0.05$; Fig. 5).

\section{The mRNA expressions of Col II and ACAN in chondrocytes}

TN14003 can significantly decrease the mRNA expressions of Col II and ACAN in chondrocytes compared to other groups. The mRNA expression levels of ACAN and Col II in the TN14003, T140, AMD3100, and blank control groups decreased in a time-dependent manner. The mRNA expression levels of ACAN and Col II were significantly higher in the TN14003 group at all-time points compared to the other three groups $(P<0.05)$; the T140 group had higher levels compared to AMD3100 group and control group $(P<0.05)$. The lowest level was detected in the control group. All data are re-shown in Fig. 6.

\section{H\&E staining and Safranin 0 staining of knee cartilage tissue in Hartley guinea pig}

We further examined the effect of TN14003, T140, and AMD3100 on articular cartilage tissues in vivo. In the TN14003 group, the basic structures of the knee cartilage (surface layer, migration layer, deep layer, and cartilage matrix calcification layer) were clear; the superficial cells were thickened, and the chondrocytes were densely arranged; the nuclei were uniformly stained, and the cartilage surface was flat; the number of cells was large. In the T140 group and AMD3100 group, the chondrocytes were densely arranged; the nuclei were uniformly stained, and the cartilage surface was flat, and the number of cells was large. In the blank control group, the superficial cells were exfoliated; cells in the migration layer were fusiform; cells in the deep and calcification layers were dissociated outward; the chondrocytes were disorderly arranged; the cartilage surface was not flat, and the nuclear staining was not uniform; the number of cells was small (Fig. $7-1$ ).

In the TN14003 group, cartilage tissue had a strong affinity to safranin 0 staining, and deep staining of the matrix was dark; proteoglycan content was obvious, and chondrocytes were evenly arranged, while the cartilage surface was more flat. In the T140 group, the chondrocytes were densely arranged, and the nuclear staining of chondrocyte was more uniform; the cartilage surface was slightly flat. In the AMD3100 group, the chondrocytes were slightly aligned, and the nuclear staining of chondrocyte was slightly uniform; the cartilage surface was slightly flat. In the blank control group, the cartilage surface was not flat, and the cartilage tissue had a reduced affinity to safranin 0 staining. The nucleus was uniformly stained, and the staining was lighter; the proteoglycan content was significantly reduced, and the cells were disorderly arranged (Fig. 7 -2). This indicated that the cartilage in the TN14003 group 
basically had no degeneration, and that in the T140 and AMD3100 groups had slight degeneration, but that in the blank control group had obvious degeneration.

\section{Results of the Modified Mankin grading system}

The Mankin histological scores of cartilage in the TN14003, T140, AMD3100, and blank control groups were successively increased, and the observed differences were statistically significant $(P<0.05)$. The degree of cartilage degeneration was the lowest in the TN14003 group (Fig. 7 - 3).

\section{Discussion}

The SDF-1/CXCR4 signaling pathway has a pivotal role in the pathological processes of $O A^{12,31}$. Previous studies have demonstrated that SDF-1 can lead to the Col II degradation of human articular cartilage, and induce the expression and secretion of MMP-3 and MMP-13 at the mRNA level ${ }^{32}$. When SDF-1 binds to CXCR4, it activates phosphorylated extracellular signal-regulated kinase (ERK) ${ }^{19,33}$, related kinases (p38MAPK) and other signaling downstream pathways ${ }^{34,35}$. In addition, it stimulates synovial membranes and chondrocytes to secrete matrix metalloproteinases (MMPs) and degrade cartilage extracellular matrix (ECM), thus promoting joint degeneration and inducing $\mathrm{OA}^{36-40}$. Our previous studies suggested that CXCR4 antagonists could regulate the cartilage degradation induced by SDF-1 by blocking the SDF-1/CXCR4 signaling pathway; increase the expression levels of Col II and ACAN in OA cartilage tissues, and promote the synthesis of $\mathrm{Col} \mathrm{II}^{15,30}$. In this experiment, chondrocytes of OA patients were treated with CXCR4 antagonists for ten days. No difference in morphology, proliferation rate, and apoptosis were observed between AMD3100, T140 and TN14003. Moreover, the results showed that TN14003, T140, and AMD3100 could reduce the secretion of MMP-3 and MMP-13 in a time-dependent manner. In addition, those drugs decreased the mRNA expression levels of ACAN and Col II decreased in a time-dependent manner; mRNA expression levels of ACAN and Col II were significantly higher, and the MMP-3 and MMP-13 were significantly lower in TN14003 group compared to other three groups (all $\mathrm{P}<$ 0.05); these levels were significantly lower in TN14003 compared to T140, and AMD3100. The observed differences might be explained with the fact that TN14003 is a derivative of T140, and thus has a higher stability and low cytotoxicity ${ }^{28,30,41}$. AMD3100, on the other hand, is a partial antagonist of CXCR4, which has low antagonistic efficiency and high cytotoxicity ${ }^{25}$.

Furthermore, we examined the effect of drugs in the Hartley guinea pig model with spontaneous OA. After 12 weeks of conventional breeding, animals were sacrificed, and articular cartilages of knees were collected and analyzed by H\&E and safranin $\mathrm{O}$ staining. The basic structures of articular cartilage were clear; chondrocytes were densely arranged; the nuclei were uniformly stained, and the cartilage surface was flat. This was not observed in the control group. Further modified Mankin score showed that the TN14003 group had the lowest score indicating that TN14003, T140, and AMD3100 could target block SDF-1/CXCR4 signaling pathway and reduce the degeneration of knee cartilage tissues in vivo. 
To sum up, our data indicated that TN14003 could decrease OA-related cartilage degeneration by blocking the SDF-1/CXCR4 signaling pathway. TN14003 showed a superior effect in protecting the cartilage from degeneration compared to T140, and AMD3100. Given its high antagonistic efficiency and good biological stability, it could be used as an effective and safe targeted drug for the prevention and treatment of OA. If TN14003 is prepared to be a biological agent for local injection in the joint, it will not only improve the effect of locally targeted therapy but also avoid the adverse effects of TN14003 systemic administration on other tissues and organs.

\section{Methods}

\section{Patients}

Thirty patients with confirmed OA (18 males and 12 females; age range: 49-70 years, mean age: $62 \pm 5.24$ years) undergoing total knee arthroplasty at First Affiliated Hospital of Kunming Medical University were recruited. Cartilage tissues from tibial and femoral osteotomy (modified Mankin score of 0 or 1 ) were collected during surgery. The study was approved by the Ethics Committee of First Affiliated Hospital of Kunming Medical University, and the informed consent was obtained from all patients.

\section{P1 chondrocytes isolation}

Chondrocytes were isolated from cartilage tissues following a previously described approach ${ }^{30}$. Briefly, after collection, cartilage tissues were cut in $1 \mathrm{~mm} \times 1 \mathrm{~mm} \times 1 \mathrm{~mm}$ size in the clean bench and were then transferred into 6-well plates. Samples were then treated with $0.25 \%$ trypsin and type II collagenase for digestion (Gibco, USA), and centrifuged in a centrifuge tube. After washing with sterile PBS, cells were seeded into a $25 \mathrm{~cm}^{2}$ culture flask ( $5 \mathrm{~mL} /$ bottle) at a cell density of $1 \times 10^{5} / \mathrm{mL}$, and cultured in a humidified incubator containing $5 \% \mathrm{CO}_{2} / 95 \%$ air at $37^{\circ} \mathrm{C}$. The medium was changed every 3 days. When cells reached $80 \%-90 \%$ in confluence, the subculture was performed.

\section{Experimental grouping}

P1 chondrocytes were randomly assigned to four groups: blank control group, TN14003 group, T140 group, and AMD3100 group. Each group was cultured in high-glucose DMEM containing 10\% fetal bovine serum and double antibody ( $100 \mathrm{U} / \mathrm{mL}$ penicillin $+0.1 \mathrm{mg} / \mathrm{mL}$ streptomycin). The TN14003 group was treated with $1000 \mathrm{nmol} / \mathrm{L}$ TN14003 and $100 \mathrm{ng} / \mathrm{mL}$ SDF-1; the T140 group with $1000 \mathrm{nmol} / \mathrm{L}$ T140 and 100ng/mL SDF-1; the AMD3100 group with 1000nmol/L AMD3100 and 100ng/mL SDF-1; only $100 \mathrm{ng} / \mathrm{mL}$ SDF-1 was added to the blank control group. All drugs were mixed in DMEM for 1 hour, and then with SDF-1 (Peprotech, USA). The cells of the four groups were cultured for 1, 2, 4, 6, 8, and 10 days. After each time point, cells were examined using MMT assay (cell viability), flow cytometry (cell apoptosis), ELISA, Quantitative real-time PCR; each method is described in detail in the following paragraph. The medium was changed every 4 days. Also, cell morphology and the number of cells were observed under an inverted phase-contrast microscope (Olympus, Japan). After treatment, cells were stained with Col II. 


\section{MTT assay}

A total of $1 \times 10^{4} / \mathrm{mL}$ cells from each group with their corresponding drugs were plated in 96 -well plates and incubated for $1,2,4,6,8$, and 10 days. After each time point, $20 \mu \mathrm{l}$ of sterile MTT dye $(5 \mathrm{mg} / \mathrm{mL}$; Amresco, USA) was added to each well and incubated for another $4 \mathrm{~h}$ at $37^{\circ} \mathrm{C}$. After removal of the medium, $200 \mu$ l of DMSO (DMSO, Amresco, USA) was added to each well and properly mixed for another $10 \mathrm{~min}$. The absorbance at $570 \mathrm{~nm}$ was determined using a microplate reader (BioTek, USA). IC50 values were calculated from the linear regression of the plot.

\section{Flow cytometry}

A total of $1 \times 10^{5} / \mathrm{mL}$ cells from each group with their corresponding drugs were plated in 6-well plates and incubated for 2, 4, 6, 8, and 10 days. After each time point, All cells were collected and treated with AnnexinV/PI staining (Invitrogen, USA). After each time point, cell were collected in the centrifuge tubes and centrifuged at $1000 \mathrm{rpm}$ for $5 \mathrm{~min}$. After that, the supernatant was discarded. Then, the cells were resuspended in PBS and the cells were resuspended in $1 \times 10^{6} / \mathrm{mL}$. The cells were centrifuged at $1000 \mathrm{rpm}$ for $5 \mathrm{~min}$. The supernatant was discarded and $5 \mu \mathrm{L}$ of the diluted $1 \times$ conjugate was pipetted. 5 $\mu \mathrm{L}$ of PI and Annexin V-FITC staining were added respectively, cells were mixed at room temperature away from light for $15 \mathrm{~min}$, then $400 \mu \mathrm{L}$ of diluted $1 \times$ liquid was added, and cells were mixedin the dark place with ice bath. Cell suspension were filtered with 300 mesh nylon mesh before detection. After detection, the testing was under $488 \mathrm{~nm}$ excitation wavelength and $525 \mathrm{~nm}$ emission wavelength was used.The apoptotic rate of the four groups was detected on a flow cytometer (Partec, Germany).

\section{ELISA}

A total of $1 \times 10^{5} / \mathrm{mL}$ cells from each group with their corresponding drugs were plated in 6-well plates and incubated for $2,4,6,8$, and 10 days. After each time point, 500ul medium was collected and quickly cryopreserved in $-80^{\circ} \mathrm{C}$ refrigerator. the concentration of MMP-3 and MMP-13 in four groups at each time point were detected by ELISA kit ( $R$ \& D Systems) according to the manufacturer's protocol, respectively. Briefly, the micro ELISA plate has been pro-coated with an antibody specific to MMP-3 and MMP-13, and the appropriate medium samples were added in each microplate well, followed with incubation for 90 min at $37^{\circ} \mathrm{C}$. After washing with wash buffer, the corresponding secondary antibody was applied. After incubation for $60 \mathrm{~min}$, the plates were washed again. The optical density was measured spectrophotometrically at a wavelength of $450 \mathrm{~nm}$. The concentration of MMP-3 and MMP-13 was calculated from a standard curve.

\section{Quantitative real-time PCR}

A total of $1 \times 10^{5} / \mathrm{mL}$ cells from each group with their corresponding drugs were plated in 6-well plates and incubated for $2,4,6,8$, and 10 days. After each time point, the total RNA was extracted using TRNzol$\mathrm{A}^{+}$Reagent Total RNA Extraction Kit and were then reversely transcribed in cDNA using a PrimeScript ${ }^{\circledR}$ RT reagent Kit Perfect Real-Time kit, following the manufacturer's instructions. After the reverse 
transcription was completed, the obtained CDNA was stored at $-80^{\circ} \mathrm{C}$ or $-20^{\circ} \mathrm{C}$ for PCR detection. The NCBI GeneBank database was searched to find the gene sequences of human Col II and ACAN. The General Biosystems (Anhui) Corporation Limited was commissioned to design and synthesize each gene primer. The primer sequence of each gene and the length of the amplified DNA fragment are shown in Table 1. The PCR reaction conditions were as follows: Stage 1: pre-denaturation, Reps: $1,95^{\circ} \mathrm{C}$ for 15 min; Stage 2: PCR reaction, Reps: $40,95^{\circ} \mathrm{C}$ for $10 \mathrm{~s}, 60^{\circ} \mathrm{C}$ for $30 \mathrm{~s}$; Stage 3: melting curve analysis, Reps: 1 , $95^{\circ} \mathrm{C}$ for $15 \mathrm{~s}, 60^{\circ} \mathrm{C}$ for $20 \mathrm{~s}, 95^{\circ} \mathrm{C}$ for $15 \mathrm{~s}$. The relative quantitative analysis method was used. The CT value represented the number of cycles at which the target amplified product reached the set threshold. The amplification fold change of the target gene in the test specimen relative to the control $=2^{-} \triangle \triangle \mathrm{CT}$. The formula was as follows: $\triangle C T=C T$ (target gene) - CT (reference gene); $\triangle \triangle C T=\triangle C T$ (experimental group)- $\triangle \mathrm{CT}$ (control group).

In this study, the blank control group in the 2-day group of the OA cartilage group was used as a reference, and the relative quantitative $2^{-\triangle \triangle C T}$ method was used, while the fold change for each group was $2^{-\triangle \triangle C T}$. Data in the experimental group were analyzed by SPSS (version 20) software. The values of each group were expressed as mean \pm standard deviation $( \pm S)$, and analysis of variance was adopted; a $=0.05$ was used as the significant level.

\section{Spontaneous OA Animals and grouping}

As the Hartley guinea pig is an accepted model for spontaneous Osteoarthritis, the Osteoarthritis Research Society International (OARSI) has developed guidelines on the histologic examination of knee joints from these animals ${ }^{42}$. So, we used Hartley guinea pigs because these animals spontaneously develop degenerative cartilage changes in the knee joint that mimic those of human OA. A total of 96 male Hartley guinea pigs, 6-month-old, were obtained from Institute of Zoology, Chinese Academy of Sciences (No. TBA287640-334). All the animals were housed in an environment with a temperature of 20$25^{\circ} \mathrm{C}$, a relative humidity of $50 \%-60 \%$, and a light/dark cycle of $12 \mathrm{hr}$. All animal studies (including the mice euthanasia procedure) were done in compliance with the regulations and guidelines of Kunming medical University animal care and conducted according to the IACUC guidelines.

Hartley guinea pigs with spontaneous OA were then randomly assigned to four groups (24 pigs/group): TN14003 group, T140 group, and AMD3100 group and control group. Four groups were subcutaneously implanted Alzet osmotic pumps into the back, and each group was pumped with TN14003, T140, and AMD3100, respectively at a concentration of $180 \mathrm{~g} / \mathrm{mL}$ daily; the blank control group was injected with saline. After 12 weeks of conventional breeding, guinea pigs were sacrificed by intraperitoneal injection with pentobarbital sodium (30 mg $/ \mathrm{kg})$, and articular cartilages from knees were collected; morphological detection of cartilage (H\&E staining and safranin $\mathrm{O}$ staining), and the Modified Mankin grading system were performed.

\section{Statistical analysis}


The data were analyzed by SPSS (version 20) software package. All data were presented as mean \pm standard deviation $( \pm S)$. The difference between groups was analyzed by Analysis of Variance, MannWhitney $U$ test, and $\chi 2$ test when applicable. A P-value of $<0.05$ was considered statistically significant.

\section{Declarations}

\section{Acknowledgements}

This study was supported by the National Natural Science Foundation of China [grant number 81960409, 81760403]; Yunnan Key projects of the Natural Science Foundation [grant number 2017FE467(-007)]; the Yunnan Province Medical Leaders Talent Project [grant number L-201601] and Expert workstation project of Shiyi Chen [grant number 2018IC102]. The funders had no role in study design, data collection and analysis, decision to publish, or preparation of the manuscript.

\section{Author contributions}

Conception and design: $\mathrm{YL}, \mathrm{KW}, \mathrm{RH}$

Analysis and interpretation of the data: ES

Drafting of the article: $Y L, K W$

Critical revision of the article for important intellectual content: $Y L, K W, R H$

Final approval of the article: All authors

Provision of study materials or patients: $G C, X Y$

Statistical expertise: $\mathrm{CH}, \mathrm{GW}$

Administrative, technical, or logistic support: $\mathrm{CH}, \mathrm{GW}$

Collection and assembly of data: ES

\section{Competing Interests Statement}

The authors declare no competing interests.

\section{Data availability}

All data generated or analyzed during this study are included in this published article.

\section{Ethics declarations}

The study was approved by the Ethics Committee of Kunming Medical University. All experiments were performed in accordance with relevant named guidelines and regulations. Informed consent was 
obtained from all participants.

\section{Consent to publish}

Not applicable.

\section{References}

1. Park, M. H., et al. FlexPro $M D \circledast$, a Combination of Krill Oil, Astaxanthin and Hyaluronic Acid, Reduces Pain Behavior and Inhibits Inflammatory Response in Monosodium lodoacetate-Induced Osteoarthritis in Rats. Nutrients.12, (2020).

2. Rim, Y. A., Nam, Y., Ju, J. H. The Role of Chondrocyte Hypertrophy and Senescence in Osteoarthritis Initiation and Progression. International journal of molecular sciences.21, (2020).

3. de Sire, A., et al. Gut-Joint Axis: The Role of Physical Exercise on Gut Microbiota Modulation in Older People with Osteoarthritis. Nutrients.12, (2020).

4. Collaborators, G. D. a. I. I. a. P. Global, regional, and national incidence, prevalence, and years lived with disability for 354 diseases and injuries for 195 countries and territories, 1990-2017: a systematic analysis for the Global Burden of Disease Study 2017. Lancet (London, England).392, 1789-1858 (2018).

5. Cleveland, R. J., Nelson, A. E., Callahan, L. F. Knee and hip osteoarthritis as predictors of premature death: a review of the evidence. Clinical and experimental rheumatology.37 Suppl 120, 24-30 (2019).

6. Wallace, I. J., et al. Knee osteoarthritis has doubled in prevalence since the mid-20th century. Proceedings of the National Academy of Sciences of the United States of America.114, 9332-9336 (2017).

7. Oo, W. M., Yu, S. P., Daniel, M. S., Hunter, D. J. Disease-modifying drugs in osteoarthritis: current understanding and future therapeutics. Expert opinion on emerging drugs.23, 331-347 (2018).

8. DeRogatis, M., et al. Non-operative treatment options for knee osteoarthritis. Annals of translational medicine.7, S245 (2019).

9. Bannuru, R. R., et al. OARSI guidelines for the non-surgical management of knee, hip, and polyarticular osteoarthritis. Osteoarthritis and cartilage.27, 1578-1589 (2019).

10. Misra, D., et al. Does knee replacement surgery for osteoarthritis improve survival? The jury is still out. Annals of the rheumatic diseases.76, 140-146 (2017).

11. Nakata, K., et al. Disease-modifying effects of COX-2 selective inhibitors and non-selective NSAIDs in osteoarthritis: a systematic review. Osteoarthritis and cartilage.26, 1263-1273 (2018).

12. Qin, H. J., et al. SDF-1/CXCR4 axis coordinates crosstalk between subchondral bone and articular cartilage in osteoarthritis pathogenesis. Bone.125, 140-150 (2019).

13. Bragg, R., et al. Stromal cell-derived factor-1 as a potential therapeutic target for osteoarthritis and rheumatoid arthritis. Therapeutic advances in chronic disease.10, 2040622319882531 (2019). 
14. Li, P., et al. Blockade of hypoxia-induced CXCR4 with AMD3100 inhibits production of OA-associated catabolic mediators IL-1 $\beta$ and MMP-13. Molecular medicine reports.14, 1475-1482 (2016).

15. Wang, K., et al. T140 blocks the SDF-1/CXCR4 signaling pathway and prevents cartilage degeneration in an osteoarthritis disease model. PloS one.12, e0176048 (2017).

16. Wei, F., et al. Attenuation of osteoarthritis via blockade of the SDF-1/CXCR4 signaling pathway. Arthritis research \& therapy.14, R177 (2012).

17. Chen, Y., et al. Attenuation of subchondral bone abnormal changes in osteoarthritis by inhibition of SDF-1 signaling. Osteoarthritis and cartilage.25, 986-994 (2017).

18. Lu, W., et al. AMD3100 Attenuates Post-Traumatic Osteoarthritis by Maintaining Transforming Growth Factor- $\beta 1$-Induced Expression of Tissue Inhibitor of Metalloproteinase-3 via the Phosphatidylinositol 3-Kinase/Akt Pathway. Frontiers in pharmacology.10, 1554 (2019).

19. Yu, T., et al. CXCR4 promotes oral squamous cell carcinoma migration and invasion through inducing expression of MMP-9 and MMP-13 via the ERK signaling pathway. Molecular cancer research : MCR.9, 161-172 (2011).

20. Mishra, R. K., Shum, A. K., Platanias, L. C., Miller, R. J., Schiltz, G. E. Discovery and characterization of novel small-molecule CXCR4 receptor agonists and antagonists. Scientific reports.6, 30155 (2016).

21. Choi, W. T., Yang, Y., Xu, Y., An, J. Targeting chemokine receptor CXCR4 for treatment of HIV-1 infection, tumor progression, and metastasis. Current topics in medicinal chemistry.14, 1574-1589 (2014).

22. De Clercq, E. Mozobil ${ }^{\circledR}$ (Plerixafor, AMD3100), 10 years after its approval by the US Food and Drug Administration. Antiviral chemistry \& chemotherapy.27, 2040206619829382 (2019).

23. Tahirovic, Y. A., et al. Small molecule and peptide-based CXCR4 modulators as therapeutic agents. A patent review for the period from 2010 to 2018. Expert opinion on therapeutic patents.30, 87-101 (2020).

24. De Clercq, E. The bicyclam AMD3100 story. Nature reviews Drug discovery.2, 581-587 (2003).

25. Zhang, W. B., et al. A point mutation that confers constitutive activity to CXCR4 reveals that T140 is an inverse agonist and that AMD3100 and ALX40-4C are weak partial agonists. The Journal of biological chemistry.277, 24515-24521 (2002).

26. De Clercq, E. The AMD3100 story: the path to the discovery of a stem cell mobilizer (Mozobil). Biochemical pharmacology.77, 1655-1664 (2009).

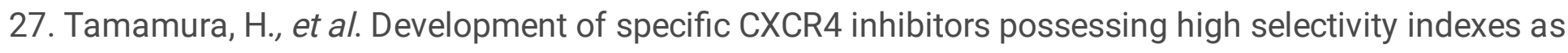
well as complete stability in serum based on an anti-HIV peptide T140. Bioorganic \& medicinal chemistry letters.11, 1897-1902 (2001).

28. Bai, R., et al. Symmetrical bis-tertiary amines as novel CXCR4 inhibitors. European journal of medicinal chemistry.118, 340-350 (2016).

29. Lefort, S., et al. CXCR4 inhibitors could benefit to HER2 but not to triple-negative breast cancer patients. Oncogene.36, 1211-1222 (2017). 
30. Jia, D., et al. miR-146a-5p expression is upregulated by the CXCR4 antagonist TN14003 and attenuates SDF-1-induced cartilage degradation. Molecular medicine reports.19, 4388-4400 (2019).

31. Wang, W., et al. Celastrol attenuates pain and cartilage damage via SDF-1/CXCR4 signalling pathway in osteoarthritis rats. The Journal of pharmacy and pharmacology.70, 81-88 (2018).

32. Kanbe, K., Takagishi, K., Chen, Q. Stimulation of matrix metalloprotease 3 release from human chondrocytes by the interaction of stromal cell-derived factor 1 and CXC chemokine receptor 4 . Arthritis and rheumatism.46, 130-137 (2002).

33. Xiang, Y., et al. miR-142-5p as a CXCR4-Targeted MicroRNA Attenuates SDF-1-Induced Chondrocyte Apoptosis and Cartilage Degradation via Inactivating MAPK Signaling Pathway. Biochemistry research international.2020, 4508108 (2020).

34. Joos, H., Albrecht, W., Laufer, S., Brenner, R. E. Differential effects of p38MAP kinase inhibitors on the expression of inflammation-associated genes in primary, interleukin-1beta-stimulated human chondrocytes. British journal of pharmacology.160, 1252-1262 (2010).

35. Lei, J., Fu, Y., Zhuang, Y., Zhang, K., Lu, D. LncRNA SNHG1 alleviates IL-1 $\beta$-induced osteoarthritis by inhibiting miR-16-5p-mediated p38 MAPK and NF-KB signaling pathways. Bioscience reports.39, (2019).

36. Wang, X. Y., Chen, Y., Tang, X. J., Jiang, L. H., Ji, P. AMD3100 Attenuates Matrix Metalloprotease-3 and -9 Expressions and Prevents Cartilage Degradation in a Monosodium lodo-Acetate-Induced Rat Model of Temporomandibular Osteoarthritis. Journal of oral and maxillofacial surgery : official journal of the American Association of Oral and Maxillofacial Surgeons.74, 927.e921-927.e913 (2016).

37. Goldring, M. B., et al. Roles of inflammatory and anabolic cytokines in cartilage metabolism: signals and multiple effectors converge upon MMP-13 regulation in osteoarthritis. European cells \& materials.21, 202-220 (2011).

38. Son, Y. O., et al. Estrogen-related receptor $y$ causes osteoarthritis by upregulating extracellular matrixdegrading enzymes. Nature communications.8, 2133 (2017).

39. Jalbă, B. A., et al. Alterations in expression of cartilage-specific genes for aggrecan and collagen type II in osteoarthritis. Romanian journal of morphology and embryology = Revue roumaine de morphologie et embryologie.52, 587-591 (2011).

40. Favero, M., et al. Inflammatory molecules produced by meniscus and synovium in early and endstage osteoarthritis: a coculture study. Journal of cellular physiology.234, 11176-11187 (2019).

41. Tamamura, H., Tsutsumi, H., Nomura, W., Tanaka, T., Fujii, N. A future perspective on the development of chemokine receptor CXCR4 antagonists. Expert opinion on drug discovery.3, 1155-1166 (2008).

42. Kraus, V. B., Huebner, J. L., DeGroot, J., Bendele, A. The OARSI histopathology initiative recommendations for histological assessments of osteoarthritis in the guinea pig. Osteoarthritis and cartilage.18 Suppl 3, S35-52 (2010).

\section{Tables}


Table 1 The sequence of primers Col II, ACAN, and hGAPDH.

\begin{tabular}{|lll|}
\hline Gene detected & PCR primer sequence (5' to $\mathbf{3}^{\prime}$ ) & DNA fragment \\
\hline Col II primer-F & AAGGATGTGTGGAAGCCGGA & 101 bp \\
\hline Col II primer-R & CTGAGGCAGTCTTTCACGTCT & \\
\hline ACAN primer-F & CTTCTGCAACTGAAGTGCCCTC & 198bp \\
\hline ACAN primer-R & GCTCTTCCGAGGCTGATGGTT & \\
\hline hGAPDH-F & TGTTGCCATCAATGACCCCT & $177 \mathrm{bp}$ \\
\hline hGAPDH-R & TCGCCCCACTTGATTTTGGA & \\
\hline
\end{tabular}

\section{Unsectioned Paragraphs}

Running title: Three CXCR4 antagonists targeted SDF-1/CXCR4 signaling pathway to attenuate cartilage degeneration

Telephone number: $+86-13529365266$

\section{Ethics declarations}

The study was approved by the Ethics Committee of Kunming Medical University.

All experiments were performed in accordance with relevant named guidelines and regulations. Informed consent was obtained from all participants.

Figures legends

\section{Figures}


A

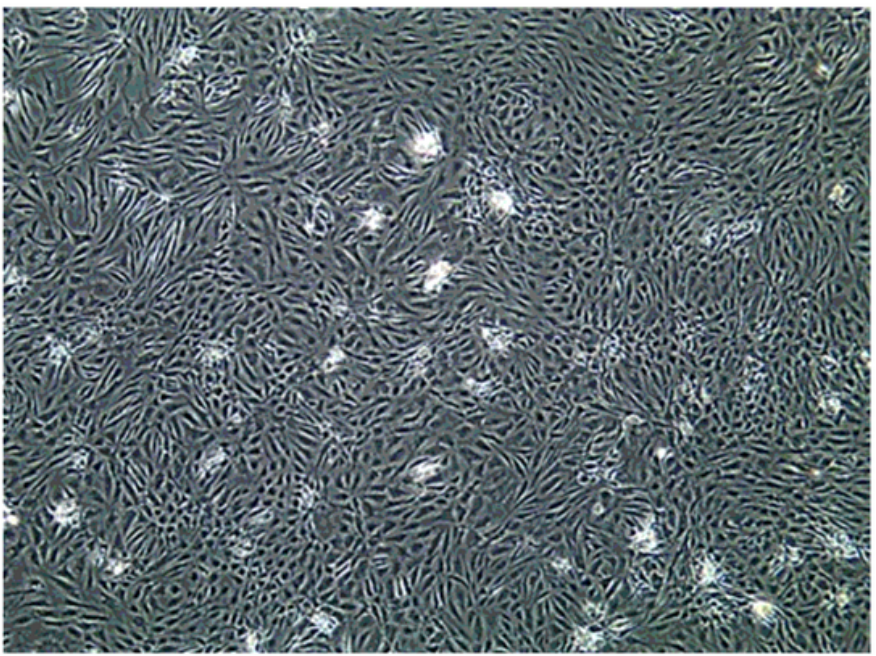

C

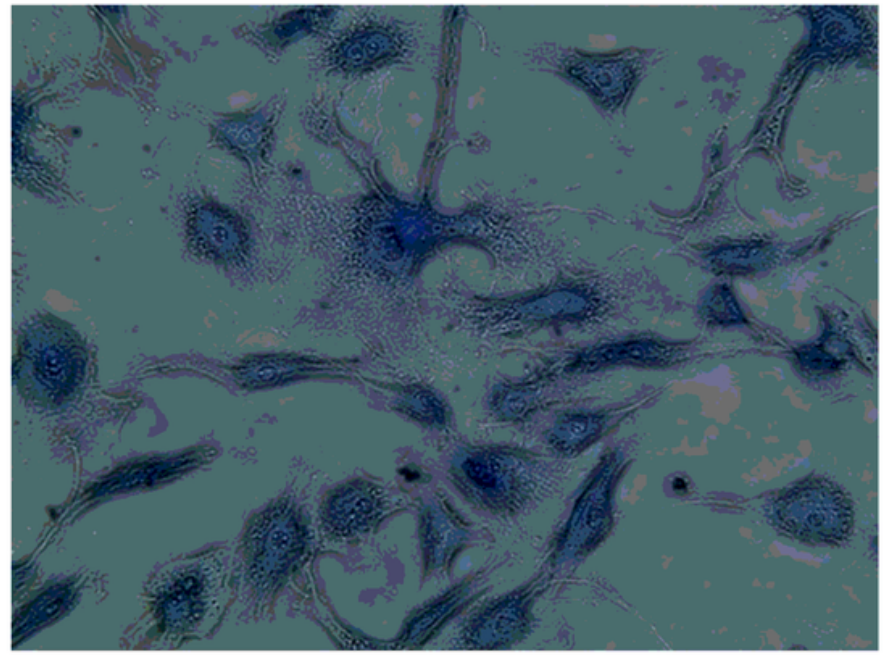

B

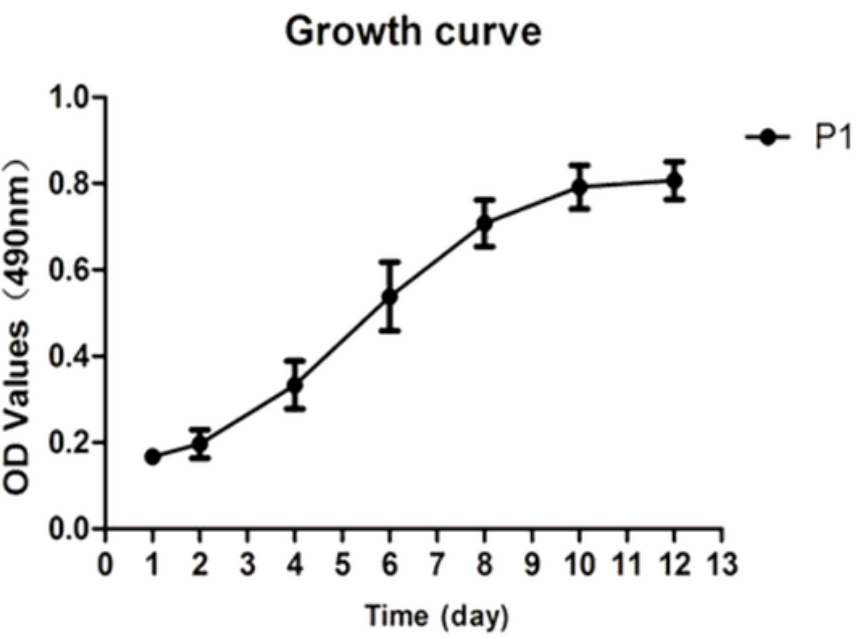

D

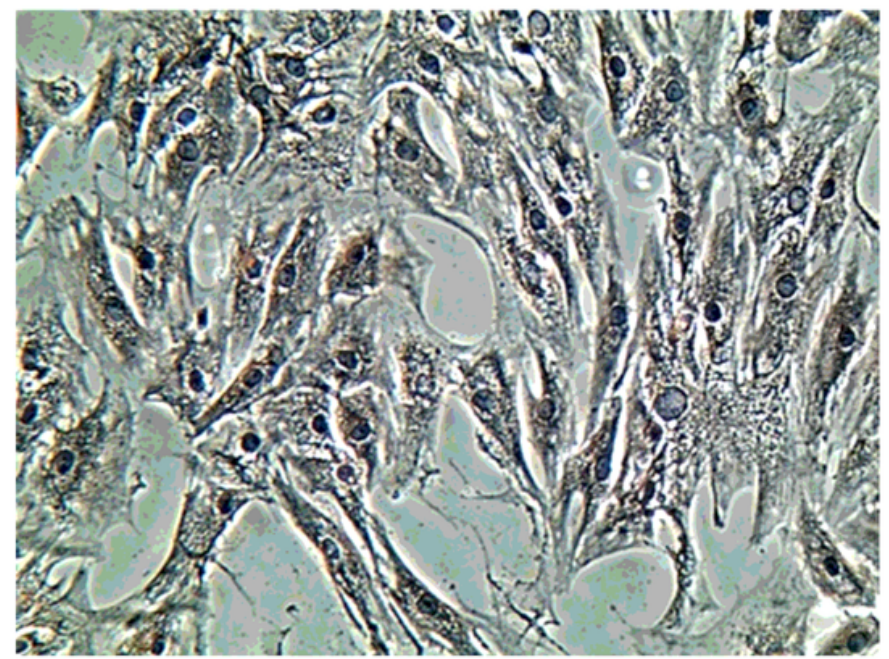

\section{Figure 1}

Morphology of primary and P1 chondrocytes of OA patients. Chondrocytes were successfully isolated from $O A$ cartilage and identified by toluidine blue staining and type II collagen staining. (a) primary chondrocytes cultured for 16 days ( $\times 40$ times). (b) The growth curve of P1 chondrocytes. (c) P1 chondrocytes were positive for toluidine blue staining ( $\times 100$ times; $\times 200$ times). (d) Col II immunohistochemical staining of cultured P1 chondrocytes $(\times 200$ times $)$. 

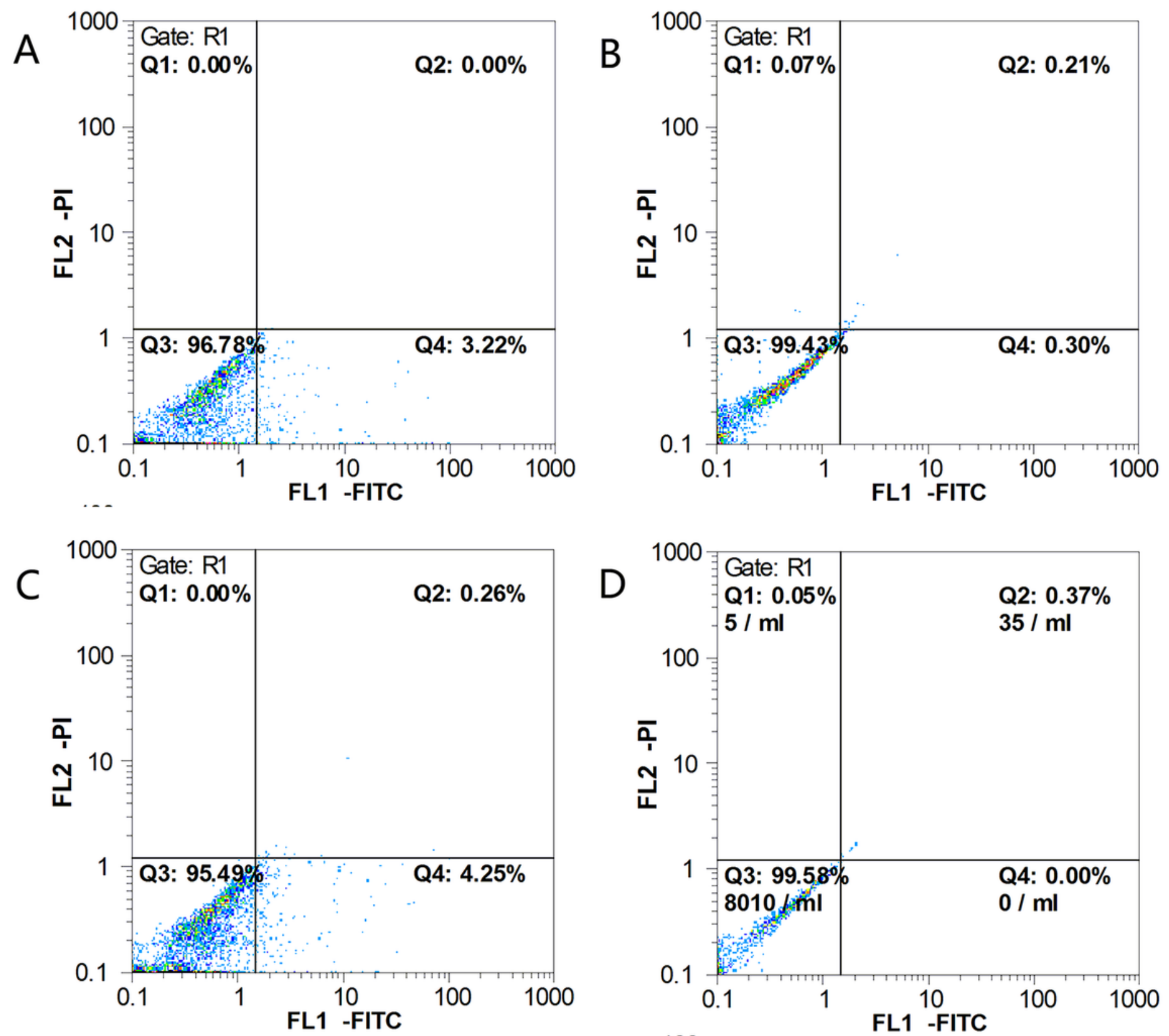

Figure 2

Apoptotic rate of P1 chondrocytes by flow cytometry. The apoptosis rate of chondrocyte was less than $5 \%$, it showed that chondrocyte was vigorous and suitable for the next experiment. (a) Annexin FITC-V staining. (b) PI along staining. (c) Annexin FITC-V and PI staining. (d) Blank control. 
Fig.3-1
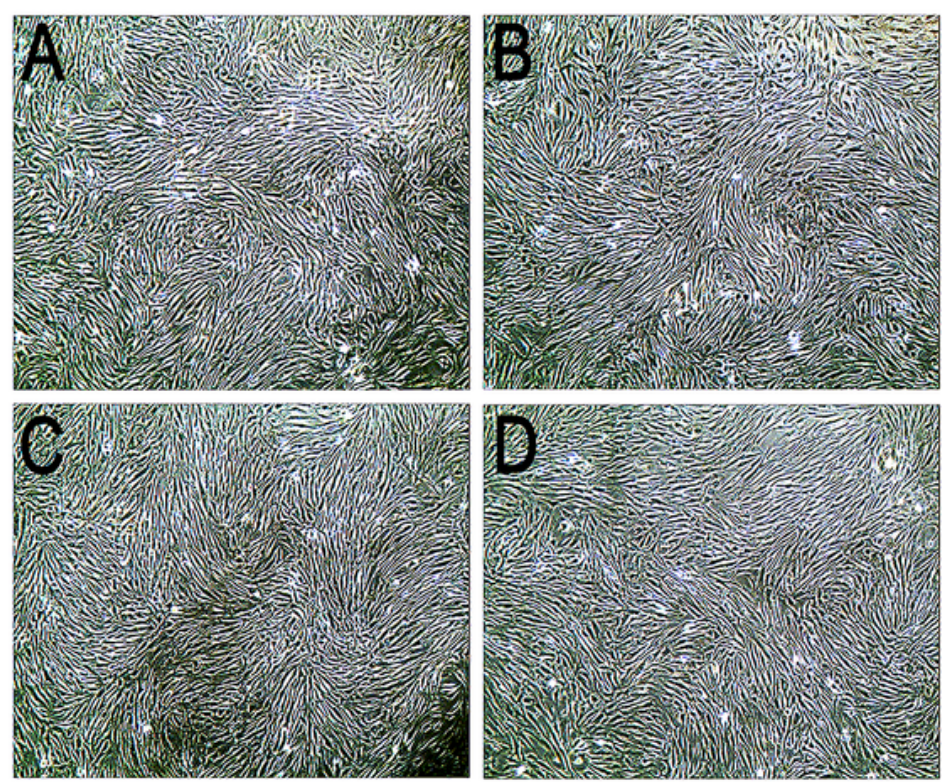

Fig.3-2
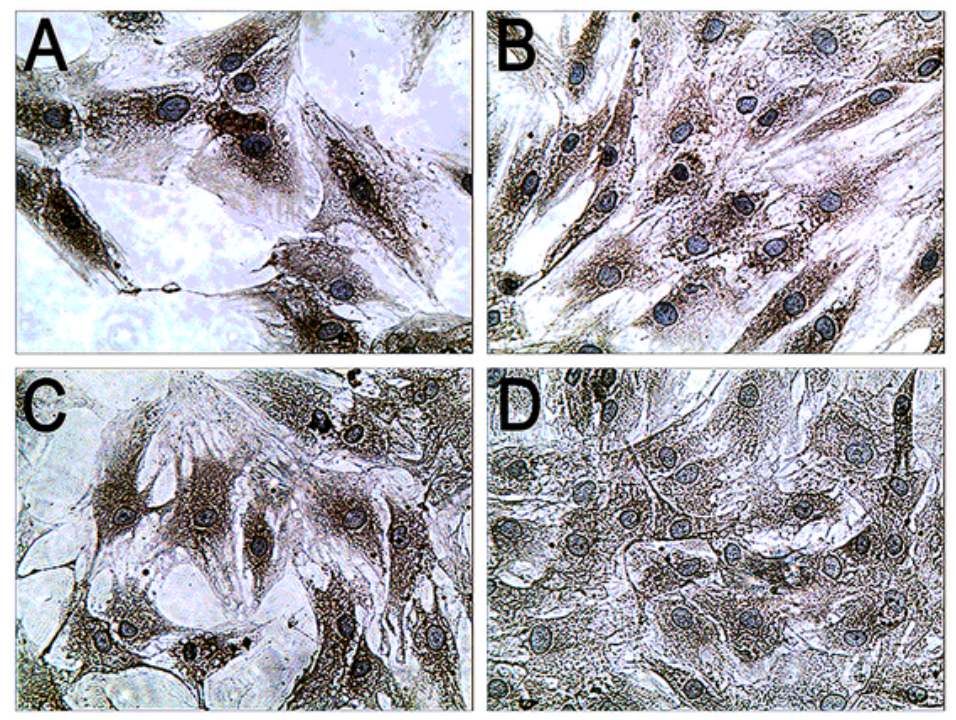

MTT

Fig.3-3

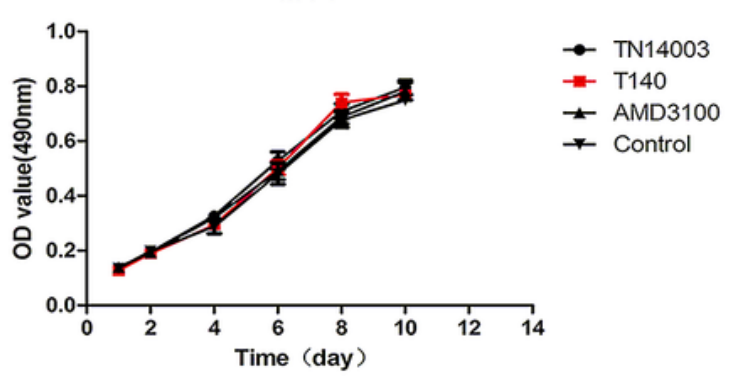

Figure 3

(3-1) At day 10 of a drug intervention, cells in the four groups showed significant spiral growth, and cell density and cell morphology were not significantly different. It showed that the three drugs (TN14003, T140 and AMD3100) have no significant effect on chondrocyte growth at 1000nmol/L concentration. (a) TN14003 group $\times 40$ times. (b) T140 group $\times 40$ times. (c) AMD3100 group $\times 40$ times. (d) Blank control group $\times 40$ times. (3-2) After 10 days of drug intervention culture, immunohistochemical staining for Col 
II. It showed that TN14003 can delay the degradation of type II collagen in chondrocyte compared to T140 and AMD3100. (a) TN14003 group $\times 200$ times. (b) T140 group $\times 200$ times. (c) AMD3100 group $\times$ 200 times. (d) Blank control group $\times 200$ times. (3-3) There was no significant difference in cell proliferation between the four groups at the same time point (MTT assay).

Fig.4-1
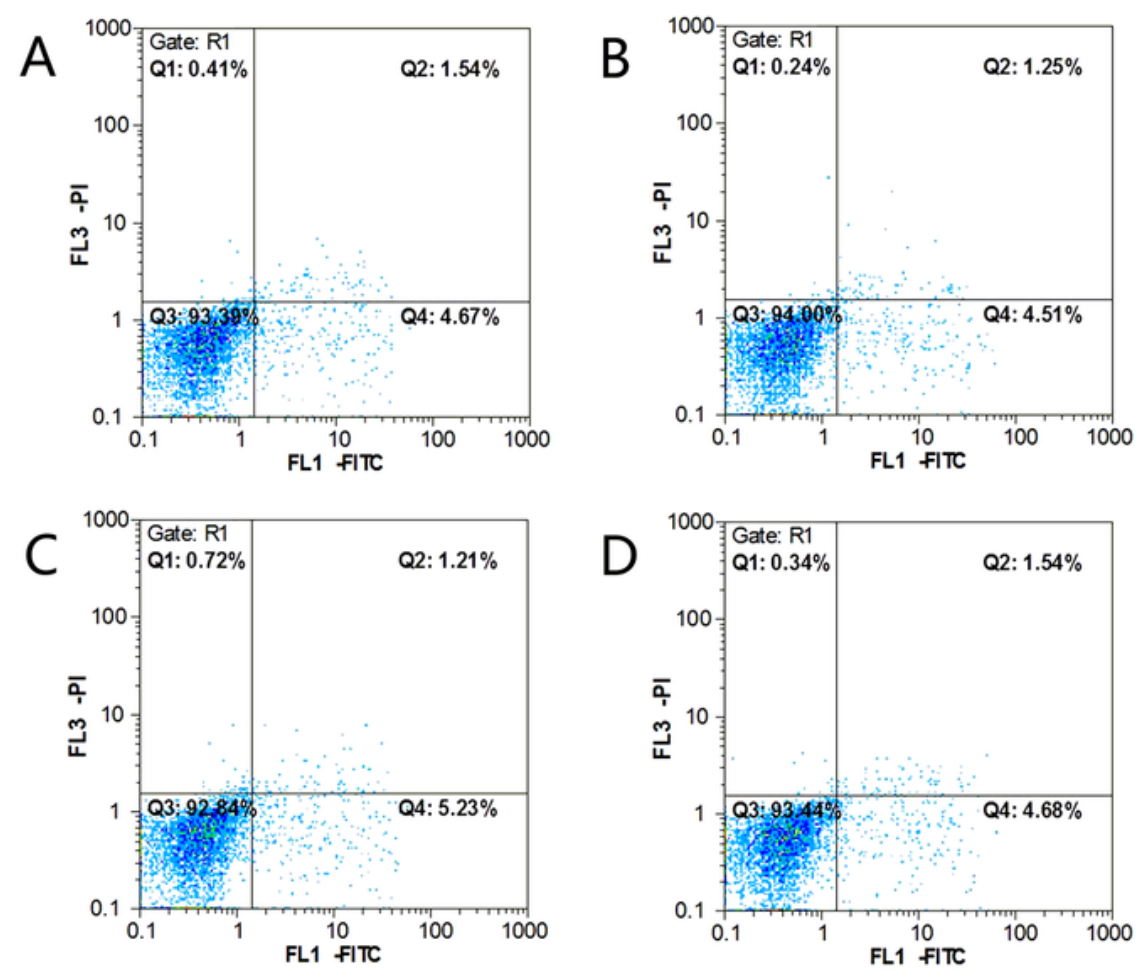

Fig.4-2

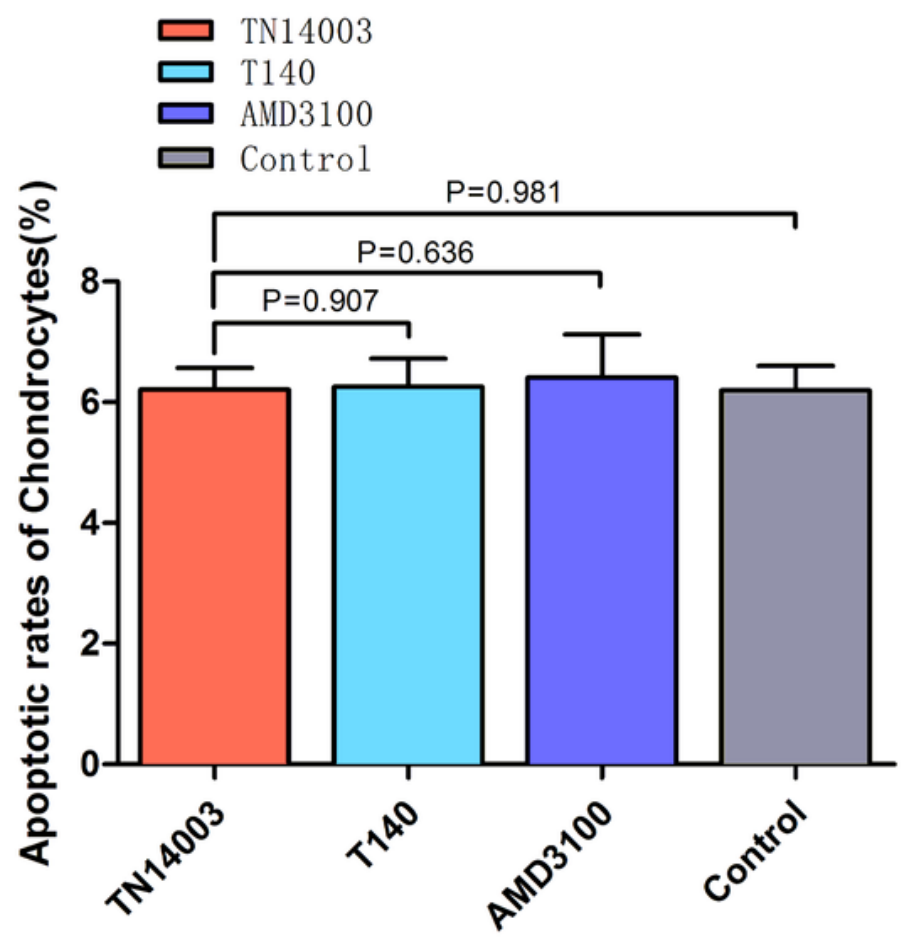

Figure 4 
Flow cytometry showed that drugs (TN14003, T140, AMD3100) at a concentration of $1000 \mathrm{nmol} / \mathrm{L}$ had no significant effect on the proliferation and apoptosis of OA chondrocytes. (4-1) Total apoptotic rate of chondrocytes flow cytometry. (a) TN14003 group. (b) T140 group. (c) AMD3100 group. (d) Blank control group. (4-2) Flow cytometry showed no significant difference in the apoptotic rate among the four groups
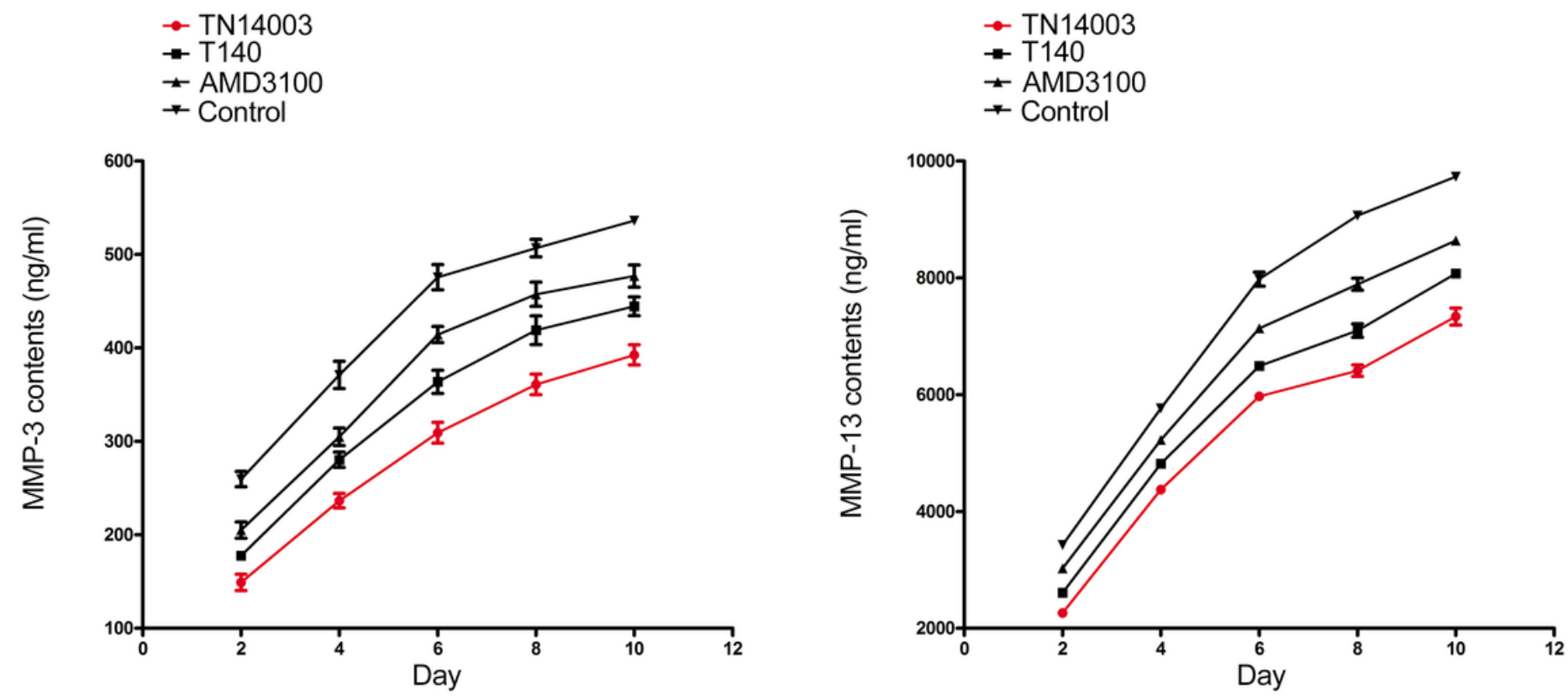

Figure 5

Detection of MMP-3 and MMP-13 content in the OA chondrocyte medium by ELISA. It showed that TN14003 can reduce the release of MMP-3 and MMP-13 in chondrocytes at all-time points compared to the other groups. 
$\begin{array}{ll}\text { Fig.6-1 } & \square \text { TN14003 } \\ & \square \text { T140 } \\ \text { AMD3100 } \\ \text { Control }\end{array}$
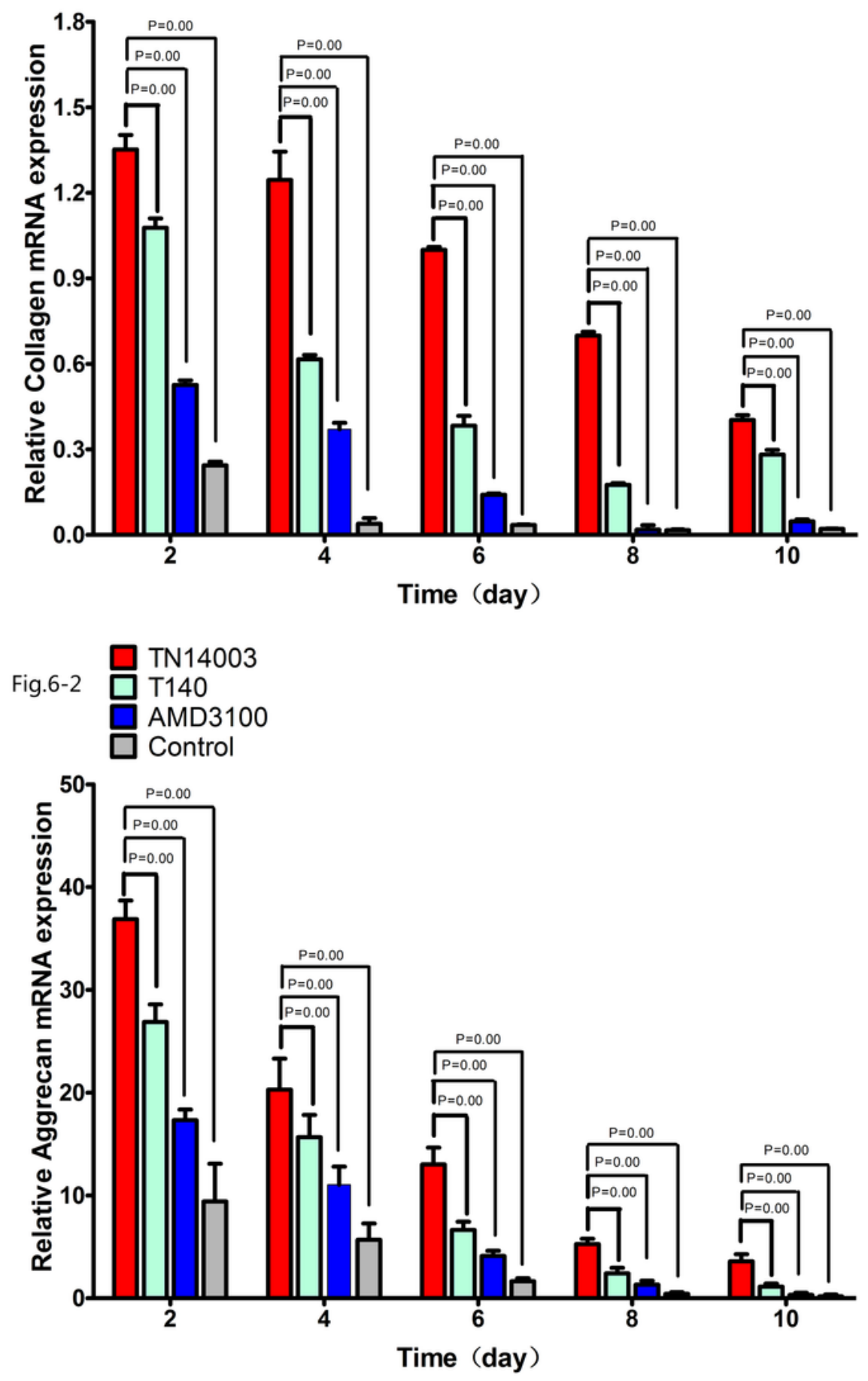

Figure 6

The mRNA expression levels of ACAN and Col II were significantly higher in the TN14003 group at all-time points compared to the other three groups. (6-1) qPCR detection of mRNA expressions of Col II in chondrocytes. (6-2) qPCR detection of mRNA expressions of ACAN in chondrocytes. 
Fig.7-1
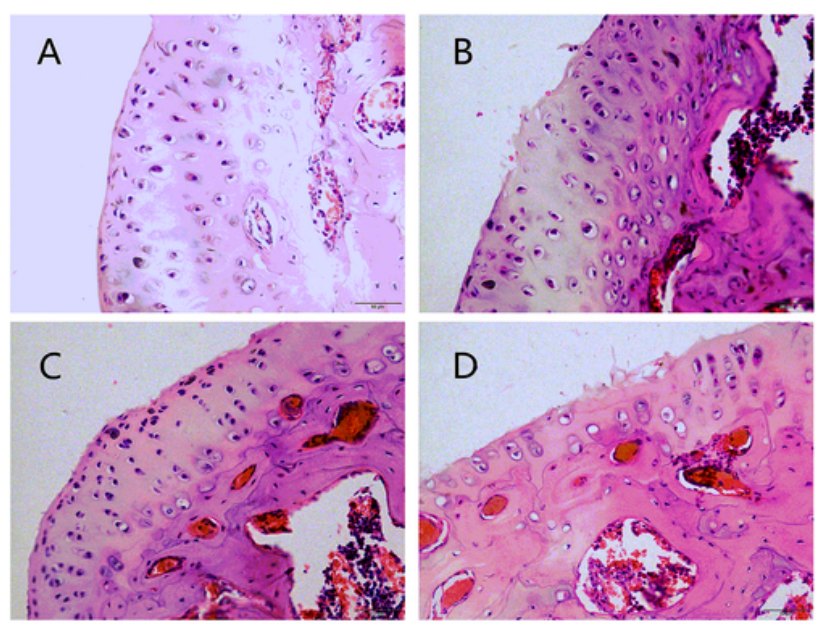

Fig.7-2

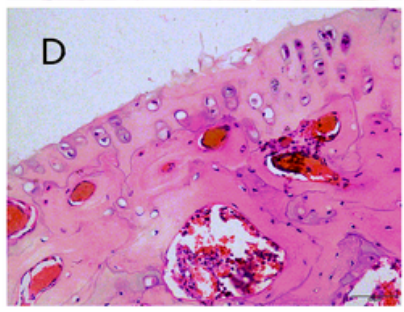

$$
\begin{array}{ll}
\square & \text { TN14003 } \\
\square & \text { T140 } \\
\square & \text { AMD3100 }
\end{array}
$$

Fig.7-3

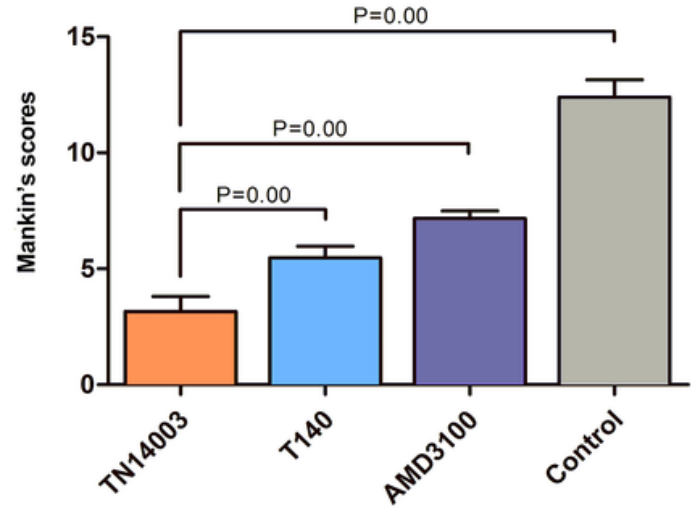

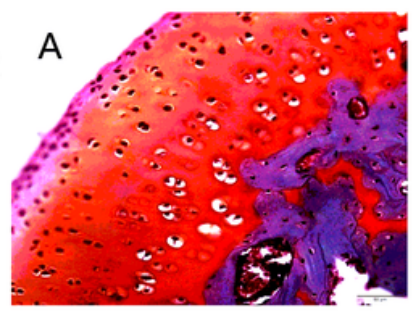
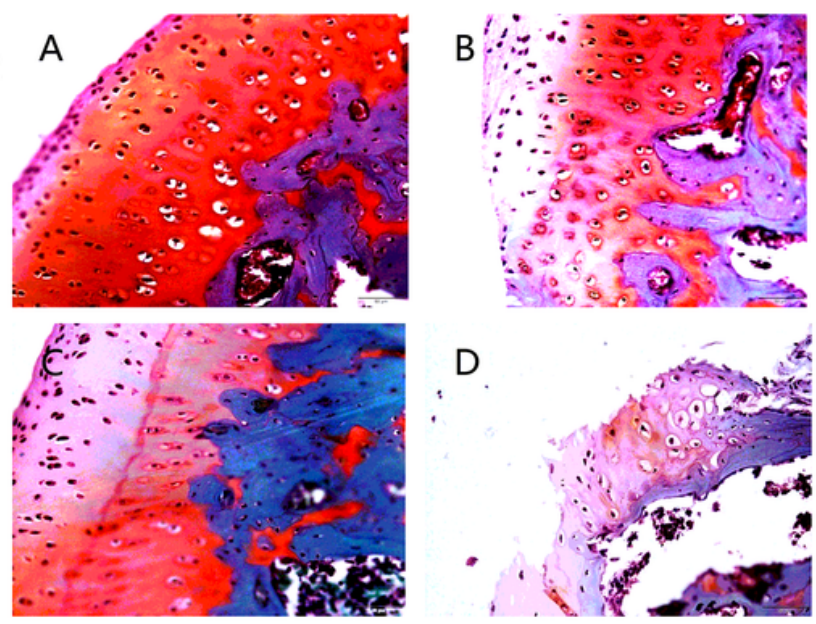

\section{Figure 7}

TN14003 can reduce the degeneration of articular cartilage. (7-1) H\&E staining of knee cartilage tissues.

(a) TN14003 group $\times 100$ times. (b) T140 group $\times 100$ times. (c) AMD3100 group $\times 100$ times. (d) Control group $\times 100$ times. (7-2) Safranin 0 staining of knee cartilage tissues. (a) TN14003 group $\times 100$ times.

(b) T140 group $\times 100$ times. (c) AMD3100 group $\times 100$ times. (d) Control group $\times 100$ times. $(7-3)$ Histogram analysis of Mankin histological score. 